\title{
The Electron Celebrates the 100th Anniversary of lts Discovery
}

The Science Museum in London and UMIST in Manchester, ${ }^{1}$ along with many other institutions, are celebrating the 100 th anniversary of the discovery of the electron. The events commemorate the publication in The Philosophical Magazine in October 1897 of Joseph John (J.J.) Thomson's discovery of corpuscles, the particles characterizing cathode rays. The term "electron" had been coined by George Johnstone Stoney, an Irish physicist, while studying Michael Faraday's Law of Electrolysis. He described a unit of electricity as "These charges, which it will be convenient to call 'electrons,' cannot be removed from the atom, but they become disguised when atoms chemically unite." Robert Andrews Millikan (the American physicist later famous for the oil drop experiment measuring electronic charge) commented that "electron" should have retained Stoney's meaning, "instead of using it to denote solely the free negative electron or corpuscle of J.J. Thomson," and Thomson continued to use the term as Stoney defined it.

Nevertheless, "electron" is the accepted term for the corpuscles that Thomson first reported to the Cambridge Philosophical Society on February 8, 1897, then to the Royal Institution as later printed in the May 21 issue of Electrician, and finally published that year as a paper, originally dated August 7, in The Philosophical Magazine.

In March of the same year, Dutch physicist Pieter Zeeman and Dutch theoretical physicist Hendrik Antoon Lorentz published an English translation of their calculation of charge to mass ratio that was close to Thomson's result. The original paper was published in Holland in $\mathbf{1 8 9 6 .}$ Walter Kaufmann also calculated the ratio of mass to charge in cathode rays in April 1897 but failed to recognize that the rays might consist of subatomic particles.

Thomson made his discovery while a professor at Cambridge at the Cavendish Laboratory, which was representative of a relatively new system of scientific research. Up until the early 19 th century, most scientists had worked as individuals or in small groups, unsupported, but by mid-century, research moved into university laboratories. At the Cavendish each

\footnotetext{
${ }_{1}$ "Life, The Universe and the Electron," April 1997-April 1998, Science Museum, South Kensington, London SW7 2DD, phone 0171938-8044, fax 0171-938-9736; "The Taming of the Electron (From the Cat's Whisker to the World Wide Web)," December 10, 1997, 2:15 p.m., Room C16, Reynold Building, UMIST, Manchester M60 1QD, phone 0161-200-3180, fax 0161-200-3941.
}

scientist arrived with a problem to study and each developed the apparatus needed for research. While researchers rarely studied together, each worked on interlocking problems. Their cameraderie and mutual respect is evident from the annual songs mathematical physicist A.A. Robb composed for the lab's Christmas parties:

My name is J.J. Thomson and my lab's in Free School Lane,

If once a man has been inside he's sure to come again,

Here some do play with Töpler pumps, and some with liquid air,

And some do play the giddy goat, but that's not here nor there.

The establishment of research in institutional laboratories saw a rapid series of discoveries including those leading up to the identification of electrons.

During Thomson's discovery, many scientists contested the idea that cathode rays were made up of subatomic particles. Even though Emil Wiechert of Germany announced in a lecture to the Scientific and Economic Society of Köningsberg, then the capital of East Prussia, at the beginning of 1897 the possibility of particles lighter than atoms, German scientists predominantly held that cathode rays were electromagnetic waves, while English scientists argued for the particle model.

Comprehensive studies of cathode rays had begun with German inventor Heinrich Geissler's production in 1855 of a vacuum pump using a column of mercury as a piston, enabling low atmospheric pressure without leakage, unlike previous vacuum pumps using leather or similar materials for the gasket or piston seals. With modifications of Geissler's pump made by Sir William Crookes of England, Julius Plücker showed how cathode rays can be produced and that they bent under the influence of a magnet, and so revolutionizing their study. More than a decade later, Cromwell Varley also concluded that cathode rays were made of particles since they bent when passing through a magnetic field. In an 1879 issue of The Philosophical Magazine, Crookes published his finding that cathode rays were made up of molecules traveling at a high speed. Crookes actually thought he had discovered a fourth state of molecules, "ultragaseous," in which the occasional collision of molecules was insignificant.

P.G. Tait, however, pointed out that if the molecules traveled rapidly, then significant shifts in wavelengths of spectral lines should have been observed, but were not.
Arthur Schuster of Manchester, also a proponent of the particle argument, accounted for the lack of the Doppler shift by saying that relatively stationary molecules struck by rapidly moving particles caused light to be emitted in the path of the cathode ray. Eugen Goldstein of Germany also countered the molecule argument, showing that cathode rays extended beyond the free mean path for molecules. He pointed out that electromagnetic rays could travel far through the glass tube filled with molecules without being scattered, whereas molecules would have collided with other molecules and been deflected.

Heinrich Hertz of Germany in 1883 erroneously determined that the cathode ray path was distinct from the path of the current, and in 1891 performed experiments to prove that the rays were some form of wave since rays could permeate thin layers of metal and atoms could not. He experimented with thin layers of gold, silver, aluminum, and also alloys of gold or silver with tin, zinc, and copper, and he experimented with successive thin layers of metals.

In the meantime, a French physics student Jean Perrin demonstrated that cathode rays carried a negative charge, a task Hertz had failed to do. Perrin placed a charge collector inside his cathode ray tube. He demonstrated that the cathode rays charged the collector. Thomson, in the experiments he published in 1897, continued with Perrin's experiment, showing that when the cathode rays were not aimed at the collector, the collector remained neutral. This experiment also disproved Hertz's argument that the rays and currents were distinct. In a second experiment, Thomson succeeded in deflecting the rays with an electric field.

In his third experiment, Thomson sought to understand what type of particles made up the cathode rays. By measuring how much a magnetic field deflected the rays and how much energy they carried, he was able to calculate the charge to mass ratio. In his Nobel Lecture presented in 1906, Thomson explained that the "carriers of negative electricity are bodies, which I have called corpuscles, having a mass very much smaller than that of the atom of any known element....The corpuscle appears to form a part of all kinds of matter under the most diverse conditions; it seems natural therefore to regard it as one of the bricks of which atoms are built up."

For Further Reading: See website http:/ / www.aip. org/history/electron. $\square$ 